\title{
AN OPTIMAL EXTENSION OF MARSTRAND'S PLANE-PACKING THEOREM
}

\author{
THEMIS MITSIS
}

\begin{abstract}
We prove that if $F$ is a subset of the 2-dimensional unit sphere in $\mathbb{R}^{3}$, with Hausdorff dimension strictly greater than 1 , and $E$ is a subset of $\mathbb{R}^{3}$ such that for each $e \in F, E$ contains a plane perpendicular to the vector $e$, then $E$ must have positive 3-dimensional Lebesgue measure.
\end{abstract}

\section{INTRODUCTION}

Marstrand [1] proved that a subset of $\mathbb{R}^{3}$ containing a translate of every plane has positive 3-dimensional Lebesgue measure. The purpose of this paper is to show that Marstrand's result still holds if one restricts the set of the normal directions of the planes to any subset of the unit sphere with Hausdorff dimension strictly greater than one. Namely, we prove the following.

Theorem. Let $S^{2} \subset \mathbb{R}^{3}$ be the 2-dimensional unit sphere, $F \subset S^{2}$ be a set of Hausdorff dimension strictly greater than 1 , and $E \subset \mathbb{R}^{3}$ be a Borel set such that for each $e \in F, E$ contains a plane perpendicular to the vector $e$. Then E has positive 3-dimensional Lebesgue measure.

We also show that this result is optimal, in the sense that the condition on the Hausdorff dimension of $F$ cannot be relaxed.

\section{Preliminaries}

$\Omega^{2}$ is the set $\left\{e \in S^{2}:|e-(1,0,0)| \leq 1 / 10\right\}$.

$B(x, r)$ is the ball of radius $r$, centered at the point $x$.

$\mathcal{L}^{k}, k=2,3$, denotes $k$-dimensional Lebesgue measure.

$\operatorname{dim}_{H}(A)$ is the Hausdorff dimension of the set $A$.

$l_{e}(a)$ is the line in the direction $e \in S^{2}$, passing through the point $a \in \mathbb{R}^{3}$.

$\Pi_{e}$ is the plane through the origin, perpendicular to the vector $e \in S^{2}$.

2000 Mathematics Subject Classification. Primary: 28A75. Secondary: 28A78.

This research has been supported by a Marie Curie Fellowship of the European Community programme "Improving Human potential and the Socio-economic Knowledge Base" under contract number HPMFCT-2000-00442. 
$\Pi_{e}^{\delta}(a)$ is the solid cylinder with center $a \in \mathbb{R}^{3}$, height $2 \delta$, radius 1 , and whose axis is in the direction $e \in S^{2}$, i.e.

$$
\Pi_{e}^{\delta}(a)=\left\{x \in R^{3}: \operatorname{dist}\left(x, l_{e}(a)\right) \leq 1, \text { and }\left|\operatorname{proj}_{l_{e}(a)}(x)-a\right| \leq \delta\right\},
$$

where $\operatorname{proj}_{l_{e}(a)}(x)$ is the orthogonal projection of the point $x$ onto the line $l_{e}(a)$.

$\chi_{A}$ is the characteristic function of the set $A$.

$A^{\delta}$ is the $\delta$-neighborhood of the set $A$, i.e

$$
A^{\delta}=\left\{x \in \mathbb{R}^{3}: \operatorname{dist}(x, A) \leq \delta\right\} .
$$

Throughout this paper, $C, C_{1}, C_{2}, \ldots$ will denote positive constants.

We finally note the following geometric fact which will allow us to control the measure of the intersection of two cylinders.

Lemma. There exists a constant $C$ such that for any $e, f \in \Omega^{2}$, and $a, b \in$ $\mathbb{R}^{3}$, we have

$$
\mathcal{L}^{3}\left(\Pi_{e}^{\delta}(a) \cap \Pi_{f}^{\delta}(b)\right) \leq C \frac{\delta^{2}}{\delta+|e-f|} .
$$

Proof. Let $\theta$ be the angle between $e$ and $f$. After a rotation and a translation, $\Pi_{e}^{\delta}(a) \cap \Pi_{f}^{\delta}(b)$ is contained in the set $\left(R_{1} \cap R_{2}\right) \times J$, where $R_{1}$ and $R_{2}$ are 2dimensional rectangles of dimensions $2 \times 2 \delta$ intersecting at angle $\theta$, and $J$ is an interval of length 2. By trigonometry, the diameter of $R_{1} \cap R_{2}$ is less than

$$
C_{1} \frac{\delta}{\delta+\sin \theta} \leq C_{2} \frac{\delta}{\delta+|e-f|} .
$$

So, $R_{1} \cap R_{2}$ is contained in a rectangle of dimensions

$$
C_{2} \frac{\delta}{\delta+|e-f|} \times 2 \delta
$$

and therefore

$$
\mathcal{L}^{2}\left(R_{1} \cap R_{2}\right) \leq C_{3} \frac{\delta^{2}}{\delta+|e-f|} .
$$

Integrating along $J$ we get that

$$
\mathcal{L}^{3}\left(\Pi_{e}^{\delta}(a) \cap \Pi_{f}^{\delta}(b)\right) \leq C \frac{\delta^{2}}{\delta+|e-f|} .
$$




\section{Proof of the Theorem}

Without loss of generality, we may assume that $F \subset \Omega^{2}$. Since $\operatorname{dim}_{H}(F)>$ 1, by Frostman's lemma (see, for example, [2]), there exists a positive, finite Borel measure $\mu$ supported in $F$ such that the quantity

$$
I_{1}(\mu):=\iint \frac{d \mu(x) d \mu(y)}{|x-y|}
$$

is finite.

Using measure theory, one can find a subset $F_{1} \subset F$ with $\mu\left(F_{1}\right)>0$ and a compact set $E_{1} \subset E$ such that

$$
\forall e \in F_{1} \exists a_{e} \in \mathbb{R}^{3}: \mathcal{L}^{2}\left(\left(\Pi_{e}+a_{e}\right) \cap B\left(a_{e}, 1\right) \cap E_{1}\right) \geq \frac{\pi}{2},
$$

and therefore

$$
\mathcal{L}^{3}\left(\Pi_{e}^{\delta}\left(a_{e}\right) \cap E_{1}^{\delta}\right) \geq \pi \delta, \forall e \in F_{1} .
$$

Now, decompose $F_{1}$ into a finite, disjoint family $\left\{A_{j}\right\}$ of sets with diameter less than $\delta$. Let $c_{j}=\mu\left(A_{j}\right)$ and pick $e_{j} \in A_{j}$. Then

$$
\begin{aligned}
\mu\left(F_{1}\right) & =\frac{1}{\delta} \sum_{j} \mu\left(A_{j}\right) \delta \leq \frac{1}{\delta} \sum_{j} c_{j} \mathcal{L}^{3}\left(\Pi_{e_{j}}^{\delta}\left(a_{e_{j}}\right) \cap E_{1}^{\delta}\right) \quad \text { (by (3.1)) } \\
& =\frac{1}{\delta} \sum_{j} c_{j} \int_{E_{1}^{\delta}} \chi_{\Pi_{e_{j}}^{\delta}\left(a_{e_{j}}\right)}=\frac{1}{\delta} \int_{E_{1}^{\delta}} \sum_{j} c_{j} \chi_{\Pi_{e_{j}}^{\delta}\left(a_{e_{j}}\right)} .
\end{aligned}
$$

By the Cauchy-Schwarz inequality

$$
\begin{aligned}
\mu\left(F_{1}\right) & \leq \frac{1}{\delta} \mathcal{L}^{3}\left(E_{1}^{\delta}\right)^{1 / 2}\left(\int\left(\sum_{j} c_{j} \chi_{\Pi_{e_{j}}^{\delta}\left(a_{e_{j}}\right)}\right)^{2}\right)^{1 / 2} \\
& =\frac{1}{\delta} \mathcal{L}^{3}\left(E_{1}^{\delta}\right)^{1 / 2}\left(\int \sum_{j, k} c_{j} c_{k} \chi_{\Pi_{e_{j}}^{\delta}\left(a_{e_{j}}\right) \cap \Pi_{e_{k}}^{\delta}\left(a_{e_{k}}\right)}\right)^{1 / 2} \\
& =\frac{1}{\delta} \mathcal{L}^{3}\left(E_{1}^{\delta}\right)^{1 / 2}\left(\sum_{j, k} c_{j} c_{k} \mathcal{L}^{3}\left(\Pi_{e_{j}}^{\delta}\left(a_{e_{j}}\right) \cap \Pi_{e_{k}}^{\delta}\left(a_{e_{k}}\right)\right)\right)^{1 / 2} .
\end{aligned}
$$

By the Lemma

$$
\begin{aligned}
\mu\left(F_{1}\right) & \leq C^{1 / 2} \mathcal{L}^{3}\left(E_{1}^{\delta}\right)^{1 / 2}\left(\sum_{j, k} \frac{c_{j} c_{k}}{\delta+\left|e_{j}-e_{k}\right|}\right)^{1 / 2} \\
& \leq C^{1 / 2} \mathcal{L}^{3}\left(E_{1}^{\delta}\right)^{1 / 2}\left(\sum_{j, k} \int_{A_{j}} \int_{A_{k}} \frac{2 d \mu(x) d \mu(y)}{|x-y|}\right)^{1 / 2} \\
& \leq\left(2 C \mathcal{L}^{3}\left(E_{1}^{\delta}\right) I_{1}(\mu)\right)^{1 / 2} .
\end{aligned}
$$


We conclude that

$$
\mathcal{L}^{3}\left(E_{1}^{\delta}\right) \geq \frac{\mu\left(F_{1}\right)^{2}}{2 C I_{1}(\mu)}
$$

for all $\delta$, and therefore $\mathcal{L}^{3}\left(E_{1}\right)>0$. Consequently $\mathcal{L}^{3}(E)>0$.

To see that the condition $\operatorname{dim}_{H}(F)>1$ cannot be relaxed, let $K \subset \mathbb{R}^{2}$ be a Kakeya set, that is, a set of planar measure zero which contains a line in every direction (see, for example, [2]), and let

$$
\begin{gathered}
F=\left\{(x, y, 0): x^{2}+y^{2}=1\right\} \subset S^{2}, \\
E=K \times \mathbb{R} .
\end{gathered}
$$

Then

$$
\operatorname{dim}_{H}(F)=1, \mathcal{L}^{3}(E)=0, \text { and } \forall e \in F \exists a_{e} \in \mathbb{R}^{3}: \Pi_{e}+a_{e} \subset E .
$$

\section{REFERENCES}

[1] J. Marstrand. Packing planes in $\mathbb{R}^{3}$. Mathematika (2) 26 (1979), 180-183.

[2] P. Mattila. Geometry of sets and measures in Euclidean spaces. Cambridge University Press, 1995.

Department of Mathematics and Statistics, University of Jyväskylä, P.O. Box 35, FIN-40351 JYvÄSKYLÄ, FINLAND

E-mail address: mitsis@maths.jyu.fi 